\title{
Características tecnológicas de la fibra de vicuñas en semicautiverio de la Multicomunal Picotani - Región Puno
}

\author{
TECHNOLOGICAL CHARACTERISTICS OF THE FIBRE OF VICUÑAS IN SEMI-CAPTIVITY OF THE \\ Multicommunal Picotani - Puno Region \\ Jesús E. Quispe Coaquiraa ${ }^{1,2,5}$, Teófilo Herrera Mamani ${ }^{3}$ Edgar Apaza Zúñiga ${ }^{1,2}$, \\ Ludwin Clavetea Quisca ${ }^{4}$, Zenón Maquera Marón ${ }^{1,2}$
}

\section{Resumen}

\begin{abstract}
El estudio tuvo como objetivo determinar la longitud de mecha y fibra, así como el diámetro de fibra descerdada de vicuñas por clase (crías, juveniles y adultos) y sexo (macho y hembra). Se realizó en el Criadero de Vicuñas en Semicautiverio de la Multicomunal Picotani, Puno, Perú, ubicado entre 4420 y $4800 \mathrm{msnm}$. Las variables respuesta se analizaron en un arreglo factorial de $2 \times 3$ conducido en un diseño completamente al azar. Los promedios de la longitud de mecha fueron $3.03 \pm 0.52$ y $2.97 \pm 0.51 \mathrm{~cm}$ para machos y hembras ( $p>0.05)$ y de $3.08 \pm 0.47,3.33 \pm 0.39$ y $2.59 \pm 0.39 \mathrm{~cm}$ en crías, juveniles y adultos, respectivamente $(p<0.05)$. Las longitudes de fibra fueron de $4.13 \pm 0.53$ y $4.06 \pm 0.55 \mathrm{~cm}$ en machos y hembras ( $>0.05)$ y de $4.22 \pm 0.28,4.57 \pm 0.27$ y $3.50 \pm 0.34 \mathrm{~cm}$ para crías, juveniles y adultas, respectivamente $(\mathrm{p}<0.05)$. Los diámetros de fibra descerdada fueron de $12.755 \pm 0.580$ y $12.719 \pm 0.398 \mu$ m para machos y hembras $(\mathrm{p}>0.05)$ y de $12.359 \pm 0.352$, $12.856 \pm 0.442$ y $12.997 \pm 0.447 \mu \mathrm{m}$ en crías, juveniles y adultas, respectivamente $(\mathrm{p}<0.05)$. Las variables en estudio no mostraron diferencias significativas para el factor sexo, en tanto que las vicuñas juveniles registraron la mayor longitud de mecha y fibra, y a las crías le correspondió la mayor finura $(\mathrm{p}<0.05)$.
\end{abstract}

Palabras clave: vicuña; fibra; Picotani; finura; longitud; mecha

\section{Abstract}

The aim of this study was to determine staple and fibre length, as well as the fibre diameter of vicuñas by age (cria, juvenile and adults) and sex (male and female). The study was carried out in the semicaptivity vicuña farm of Multicomunal Picotani, Puno,

\footnotetext{
${ }^{1}$ Facultad de Medicina Veterinaria y Zootecnia, Universidad Nacional del Altiplano - UNA, Puno, Perú

${ }^{2}$ Instituto de Investigación y Promoción de los Camélidos Sudamericanos IIPC-UNA, Puno, Perú

${ }^{3}$ Dirección Regional Agraria, Puno, Perú

${ }^{4}$ Centro de Innovación Tecnológica Alpaca - CITE, Puno, Perú

${ }^{5}$ E-mail: jesusquispecoaquira@gmail.com
}

Recibido: 6 de marzo de 2017

Aceptado para publicación: 26 de agosto de 2017 
Peru, located between 4420 and $4800 \mathrm{~m}$ above the sea level. Response variables were analyzed in a factorial arrangement of $2 \times 3$ conducted in a completely radomized design. The mean staple length was $3.03 \pm 0.52$ and $2.97 \pm 0.51 \mathrm{~cm}$ for males and females $(p>0.05)$ and $3.08 \pm 0.47,3.33 \pm 0.39$ and $2.59 \pm 0.39 \mathrm{~cm}$ for crias, juveniles and adults respectively $(\mathrm{p}<0.05)$. The fibre length was $4.13 \pm 0.53$ and $4.06 \pm 0.55 \mathrm{~cm}$ in males and females $(\mathrm{p}>0.05)$ and $4.22 \pm 0.28,4.57 \pm 0.27$ and $3.50 \pm 0.34 \mathrm{~cm}$ for crias, juveniles and adults respectively $(\mathrm{p}<0.05)$. The fibre diameter was $12.755 \pm 0.580$ and $12.719 \pm 0.398 \mathrm{im}$ for males and females $(\mathrm{P}>0.05)$, and $12.359 \pm 0.352,12.856 \pm 0.442$ and $12.997 \pm 0.447 \mu \mathrm{m}$ for juveniles and adults, respectively $(\mathrm{p}<0.05)$. The variables in the study did not show significant differences for the sex factor, while the juvenile vicuñas recorded the greatest staple and fibre length, and the crias had the greatest fineness $(\mathrm{p}<0.05)$.

Key words: vicuña; fiber; Picotani; fineness; length; staple

\section{INTRODUCCIÓN}

La vicuña (Vicugna vicugna) es la especie más pequeña de los camélidos sudamericanos (CSA) andinos silvestres, que junto a los domésticos, pastorea las extensas praderas altoandinas del Perú, ubicados por encima de $\operatorname{los} 3800 \mathrm{~m}$ de altitud. La vicuña produce la fibra más fina del mundo (10 a $12 \mu \mathrm{m})$ (Quispe et al., 2015), que unida a sus excelentes cualidades de suavidad al tacto, brillo, poder calorífico y color natural insustituible es muy cotizada en el mercado textil para la fabricación de telas de alta calidad (Hoffman et al., 1983). En el país, dada la población existente y el alto precio de su fibra, es la especie con mayor potencial económico para las regiones de Ayacucho, Puno, Huancavelica y Junín (DGFFS, 2014).

El Perú llegó a tener 5-10 mil vicuñas en 1964, estando cerca de la extinción de la especie, pero pasó a tener una población de 61042 ejemplares en 1986 y el último Censo Nacional registró 208899 vicuñas (DGFFS, 2014). A partir de 1996, el Estado Peruano implementó la utilización de Módulos de Uso Sustentable (MUS) como un programa de manejo en cautiverio (hoy a través de declaraciones de manejo) de las vicuñas para facilitar el monitoreo y reducir su caza furtiva (DGFFS, 2012).

La región Puno, después de Ayacucho (62 133 vicuñas), posee la mayor población de vicuñas (38 673), pero lleva el liderazgo en cuan- to al número de organizaciones sociales $(\mathrm{n}=212)$ dedicadas al manejo y conservación de la especie (DGFFS, 2012)

La fibra de las vicuñas se obtiene a través de la técnica ancestral prehispánica denominada Chacu. En la actualidad es una actividad comunal anual que convoca la participación de muchas personas, quienes forman un cerco, caminan y arrean para capturar y esquilar a las vicuñas silvestres (Quispe et al., 2015). Empero, el manejo y conservación de vicuñas está regulada por un marco legal por su condición de especie en peligro de extinción (DGFFS, 2012). La mayor proporción de vicuñas capturadas en el Chacu 2014 de la Región Puno se produjo en la puna húmeda (84\%), donde la mayoría se cría bajo un sistema de semicautiverio. En esta región, la provincia San Antonio de Putina destaca por la mayor población de vicuñas (11 200) y el mayor volumen de vicuñas capturadas (53\%) (Quispe et al., 2015).

La finura constituye el principal criterio de clasificación y de fijación del precio de la fibra del vellón de la vicuña; la cual, junto a la longitud de mecha y fibra, determinan el rendimiento del procesado y el uso final de la fibra (Quispe et al., 2015). Debido a ello, no solo es importante determinar las características físicas de 
la fibra a través de métodos rápidos, precisos y eficientes, sino determinar su variabilidad en función al sexo y la clase animal de la cual proviene. Asimismo, es importante determinar la uniformidad del diámetro y la longitud de fibra, como parte de la calidad de la fibra, siendo aceptable una variación del 5\% en la finura (Butler y Dolling, 1995).

Bajo el sistema de manejo y conservación en semi-cautiverio, es raro capturar vicuñas que pertenezcan al mismo grupo de un Chacu al siguiente; o sea, la esquila de animales involucra diferentes clases e intervalos de crecimiento de la fibra. Por ello es importante identificar los valores medio, mínimo y máximo de la finura y longitud de fibra requeridos para su posterior procesamiento industrial. Las investigaciones realizadas (Calle, 1982; Montesinos, 2000; Quispe et al., 2009; Zavaleta et al., 2009) solo incluyen la información de vicuñas juveniles y adultas que indican que el diámetro y longitud de mecha no se afecta por el factor sexo, y que la finura y la longitud de fibra disminuyen con la edad; pero no se dispone de suficiente información sobre las crías, a excepción del estudio de Bravo (1977).

El escaso conocimiento de las principales características físicas de la fibra de vicuña descerdada, a nivel de la Multicomunal Picotani (antes SAIS Picotani) de la provincia de San Antonio de Putina, ha conllevado a que los comerciantes monopólicos utilicen argumentos subjetivos para reducir el precio de la fibra y, por consiguiente, los ingresos económicos de los criadores. En virtud a ello, se ha planteado el presente trabajo de investigación para determinar el diámetro y la longitud de mecha y fibra descerdada de vicuñas y sus variabilidades por clase animal y sexo, las cuales contribuirán a mejorar la capacidad de negociación de los criadores para lograr precios justos; además de servir de referencia para formulación de normas técnicas racionales sobre clasificación de la fibra de vicuñas del Perú.

\section{Materiales y Métodos}

\section{Lugar del Estudio}

El trabajo de investigación se realizó en el Criadero de Vicuñas en Semicautiverio de la Multicomunal Picotani, del distrito de Putina, provincia de San Antonio de Putina, Región Puno, Perú. La Reserva Natural está ubicada entre 4420 y $4800 \mathrm{msnm}$, al noreste de la ciudad de Puno. La zona presenta una precipitación media anual de $670 \mathrm{~mm}$, donde el $70-80 \%$ ocurre entre noviembre y abril, una temperatura media anual de $2.6^{\circ} \mathrm{C}$, con una máxima de $19.5^{\circ} \mathrm{C}$ y una mínima de $-11.4{ }^{\circ} \mathrm{C}$ (SENAMHI, 2012). Las temperaturas mas bajas se presentan entre junio y agosto.

\section{Condiciones Ecológicas}

En las praderas naturales predominan las agrupaciones de gramíneas: Calamagrostis vicunarum (crespillo), Festuca dolichophylla (chilligua), Stipa ichu (ichu), Stipa obtusa (tisña ichu), Distichlis humilis (grama), Distichia muscoides (kunkuna), Alchemilla pinnata (sillusillu), Hipochoeris sp (pilli), Gentianella peruviana (jallu jallu), Trifolium amabili (layo). En los pastizales de laderas durante la época de lluvias predominan Stipa brachyphylla, Sitpa ichu, Muhlembergia fastigiata, Alchemilla pinnata, Bromus unioloides y Festuca rigescens; y en pastizales húmedos (bofedales) predominan Distichia muscoides, Festuca dolichophylla, Hipochoeris setenocephala, Calamagrostis crysantha, Calamagrostis brevifolia, Scirpus rigidus y Eleocharis albibracteata (Deza, 1988). Entre otras especies de importancia están especies de las familias Compositae, Caryophyllaceae, Malvaceae y Geraniaceae.

El sistema hídrico del área está formado por manantiales y pequeños riachuelos que se originan en las laderas de algunos cerros por filtración del agua de lluvia. 


\section{Sistema de Crianza}

Desde 1985, la Multicomunal Picotani mantiene un sistema de crianza de vicuñas en semi-cautiverio dentro de un cerco perimétrico de alambrado y en una extensión de 2053 ha. Las vicuñas pastorean libremente vigilados por un guardaparques. La Multicomunal está integrada por 320 socios pertenecientes a los comités conservacionistas de las comunidades de Picotani, Toma y Cambría, que en conjunto tienen más de 3500 vicuñas. Coordina con el Centro de Innovación Tecnológica de Camélidos Sudamericanos (CITECS), con sede en la ciudad de Juliaca, para el servicio de capacitación y asistencia en la esquila de vicuñas y con la División de Camélidos Sudamericanos (ex Consejo Nacional de Camélidos Sudamericanos) de la Dirección Regional de Agricultura, Puno, que autoriza la realización del Chacu.

Las normas legales sobre la conservación y explotación racional de la vicuña establecen que el régimen de propiedad y comercialización de la fibra de vicuña se da por el uso de las praderas, que son propiedad de la Multicomunal. Los socios tienen la autorización del aprovechamiento sostenible del recurso zoogenético, bajo reglamentación y supervisión del Estado, que se concretiza en la venta de la fibra de vicuña.

\section{Chacu de Vicuñas}

Se realiza anualmente para la esquila, descerdado y comercialización de la fibra. Se elige una época sin lluvias ni nevadas. Se inicia a tempranas horas del día apoyados por grupos estratégicamente ubicados en el terreno. El cerco humano arrea las vicuñas hacia la manga de captura y se las encierra en el corral de espera.

Al día siguiente, se hace la identificación crotal de las crías y se les libera sin esquilar. Asimismo, se apartan de la esquila a las vicuñas adultas con fibras cortas o con caspa, así como las vicuñas viejas, débiles, enfermas o con gestación avanzada.
Se esquila alrededor del $30 \%$ de la población, siguiendo las Buenas Prácticas de Esquila que proporciona el CITECS. Aquelas con fibra corta $(<2 \mathrm{~cm})$ se dejan libres para la esquila del próximo año. Al concluir la esquila, todas las vicuñas se liberan al campo.

En la Multicomunal Picotani, para propósitos del presente estudio, la medición de la longitud de mecha de las crías no se realiza al momento de nacer, sino cuando tienen 8-10 meses de edad; las juveniles se esquilan con 20 meses de crecimiento de fibra, aproximadamente (se incluye la longitud lograda durante la gestación); y en las adultas la esquila se realiza casi bianualmente, siempre que presenten una longitud de mecha mínima de $2.5 \mathrm{~cm}$.

\section{Animales y Muestras}

La longitud de mecha se midió, previo a la esquila, con una regla graduada, y a la vez, se tomó una muestra de mechas de fibra (4 g) de la zona del costillar medio. En el caso de las crías, la muestra se obtuvo al momento del aretado.

Se tomaron muestras de fibra de 96 vicuñas, en proporcionanes similares por sexo (macho y hembra) y clase (crías, juveniles y adultos). Las muestras se colocaron en una bolsa de polipropileno rotulado para identificar el sexo y la clase de vicuñas.

\section{Análisis de Laboratorio}

Las muestras de fibra de vicuña se analizaron en el Laboratorio de Fibras del Centro de Innovación Tecnológica de Camélidos Sudamericanos (CITECS), con sede en la ciudad de Juliaca.

Con ayuda de una lupa, se procede al descerdado de las muestras, el cual consiste en la extracción manual de los pelos o fibras meduladas que afloran a la superficie de la mecha. Luego se abre la mecha para extraer los pelos entrecruzados, siguiendo el procedimiento establecido por la Norma Técnica del IBNORCA (1988). 
Para medir la longitud de mecha y la longitud de fibra se siguieron los procedimientos indicados en la Norma Técnica Peruana (NTP) 231.304-2004 del IPAC (2004). Asimismo, para el acondicionamiento y lectura de muestras para determinar el diámetro de fibra descerdada se siguieron los procedimientos de la NTP 231.098-2005 (IPAC, 2005).

\section{Análisis Estadístico}

Para la descripción de las variables respuesta se recurrió a las medidas de tendencia central (promedio) y de dispersión (desviación estándar, coeficiente de variabilidad y amplitud o valores extremos). Para el análisis estadístico se utilizó un arreglo factorial de $2 \times 3$ con 16 repeticiones bajo un Diseño Completo al Azar. La comparación de los promedios de las variables respuesta se realizó a través de la Prueba de Duncan a un $\alpha=0.05$.

\section{Resultados}

\section{Longitud de Mecha}

En el Cuadro 1 se presentan los valores de longitud de mecha, según sexo y clase animal. El promedio general de la longitud de mecha fue $3.00 \pm 0.52 \mathrm{~cm}$, con una variabilidad de $17.20 \%$ y valores extremos entre 2.00 y $3.93 \mathrm{~cm}$, sin diferencia significativa entre sexos. La mayor longitud de mecha correspondió a las vicuñas juveniles, seguida de las crías y las adultas $(\mathrm{p}<0.05)$.

\section{Longitud de Fibra}

En el Cuadro 2 se presentan los valores de longitud de fibra, según sexo y clase animal. El promedio general de la longitud de fibra fue $4.10 \pm 0.54 \mathrm{~cm}$, con una amplitud de 2.80 a $5.27 \mathrm{~cm}$, sin diferencia significativa entre sexos. Con relación a la clase, la mayor longitud de mecha correspondió a las vicuñas juveniles, seguida de las crías y las adultas $(p<0.05)$. Asimismo, se observa una mayor variabilidad de longitud de fibra en vicuñas adultas $(9.81 \%)$, seguida de crías (6.61\%) y juveniles (5.89\%).

\section{Diametro de Fibra}

El promedio general del diámetro de fibras descerdadas de las vicuñas fue $12.77 \pm$ $0.52 \mu \mathrm{m}$, con una amplitud de 11.67 a 14.15 $\mu \mathrm{m}$, y una variabilidad del $4.54 \%$ (Cuadro 3). El factor sexo no influyó significativamente, mientras que las fibras de las crías presentaron el menor diámetro, siendo estadísticamente diferente al diámetro de las fibras de las vicuñas juveniles y adultas $(\mathrm{p}<0.05)$.

\section{Discusión}

\section{Longitud de Mecha}

La longitud de mecha, después de la finura, es la característica que sigue en importancia por su contribución en el peso de vellón y constituir un parámetro clave en la industria textil (Quispe et al., 2016). Desde el punto de vista comercial, el término longitud se refiere a la mecha; es decir, el promedio de la longitud desde la base de la mecha hasta las fibras más largas. El largo de mecha (grupo de fibras) no solo se utiliza para clasificar a los vellones como de mecha corta o larga, sino que, en las vicuñas se utiliza como criterio básico para determinar si se esquila al animal.

La longitud de mecha de las vicuñas de Picotani, para el factor sexo, se ha sostenido con el tiempo (Deza, 1988 [hembras: $3.09 \mathrm{~cm}$; machos: $3.04 \mathrm{~cm}$ ]), pero a la vez, se encuentra por encima del promedio regional de vicuñas en semicautiverio (Aguilar, 2009 [hembras: $2.93 \mathrm{~cm}$; machos: $2.90 \mathrm{~cm}$ ]). No obstante, a nivel de la Región Puno resalta la mayor longitud de mecha de las vicuñas de Cala Cala (Bravo, 1977 [hembras: $3.43 \mathrm{~cm}$; machos: $3.36 \mathrm{~cm}$ ]; Jahuira (1982) [hembras: $4.62 \mathrm{~cm}$; machos: $4.94 \mathrm{~cm}$ ]). Asimismo, las vicuñas de la región de Huancavelica tien- 
Cuadro 1. Longitud de mecha $(\mathrm{cm})$ de vicuñas por sexo y clase animal

\begin{tabular}{lcccc}
\hline Factor & N. $^{\circ}$ & Promedio $\pm \mathrm{DE}$ & $\mathrm{CV}(\%)$ & Valores extremos \\
\hline Sexo & & & & \\
Machos & 48 & $3.03 \pm 0.52^{\mathrm{a}}$ & 17.25 & $2.05-3.93$ \\
$\quad$ Hembras & 48 & $2.97 \pm 0.51^{\mathrm{a}}$ & 17.27 & $2.00-3.90$ \\
Clase & & & & \\
Cría & 32 & $3.08 \pm 0.47^{\mathrm{b}}$ & 15.33 & $2.28-3.78$ \\
Juvenil & 32 & $3.33 \pm 0.39^{\mathrm{a}}$ & 11.60 & $2.68-3.93$ \\
Adulto & 32 & $2.59 \pm 0.39^{\mathrm{c}}$ & 15.08 & $2.00-3.53$ \\
\hline Total & 96 & $3.00 \pm 0.52$ & 17.20 & $2.00-3.93$ \\
\hline
\end{tabular}

$a, b, c$ Superíndices diferentes dentro de columnas indican diferencia estadística $(p<0.05)$

$\mathrm{DE}=$ Desviación estándar; $\mathrm{CV}=$ Coeficiente de variabibilidad

Cuadro 2. Longitud de fibra $(\mathrm{cm})$ de vicuñas por sexo y clase animal

\begin{tabular}{lcccc}
\hline Factor & N. $^{\circ}$ & Promedio \pm DE & CV (\%) & Valores extremos \\
\hline Sexo & & & & \\
Machos & 48 & $4.13 \pm 0.53^{\mathrm{a}}$ & 12.96 & $3.16-5.27$ \\
$\quad$ Hembras & 48 & $4.06 \pm 0.55^{\mathrm{a}}$ & 13.46 & $2.80-5.03$ \\
Clase & & & & \\
Cría & 32 & $4.22 \pm 0.28^{\mathrm{b}}$ & 6.61 & $3.60-4.66$ \\
Juvenil & 32 & $4.57 \pm 0.27^{\mathrm{a}}$ & 5.89 & $3.95-5.27$ \\
Adulto & 32 & $3.50 \pm 0.34^{\mathrm{c}}$ & 9.81 & $2.80-4.15$ \\
\hline Total & 96 & $4.10 \pm 0.54$ & 13.16 & $2.80-5.27$ \\
\hline
\end{tabular}

$a, b, c$ Superíndices diferentes dentro de columnas indican diferencia estadística $(p<0.05)$

$\mathrm{DE}=$ Desviación estándar $; \mathrm{CV}=$ Coeficiente de variabibilidad

Cuadro 3. Diámetro de fibra $(\mathrm{cm})$ de vicuñas por sexo y clase animal

\begin{tabular}{lcccc}
\hline Factor & N. $^{\circ}$ & Promedio $\pm \mathrm{DE}$ & $\mathrm{CV}(\%)$ & Valores extremos \\
\hline $\begin{array}{l}\text { Sexo } \\
\text { Machos }\end{array}$ & 48 & $12.755 \pm 0.580^{\mathrm{a}}$ & 4.543 & $11.673-14.150$ \\
$\quad$ Hembras & 48 & $12.719 \pm 0.398^{\mathrm{a}}$ & 3.133 & $11.815-13.557$ \\
Clase & & & & \\
Cría & 32 & $12.359 \pm 0.352^{\mathrm{b}}$ & 2.845 & $11.815-12.942$ \\
Juvenil & 32 & $12.856 \pm 0.442^{\mathrm{a}}$ & 3.437 & $11.673-13.557$ \\
Adulto & 32 & $12.997 \pm 0.447^{\mathrm{a}}$ & 3.439 & $12.229-14.150$ \\
\hline Total & 96 & $12.771 \pm 0.516$ & 4.543 & $11.673-14.150$ \\
\hline
\end{tabular}

$a, b, c$ Superíndices diferentes dentro de columnas indican diferencia estadística $(p<0.05)$

$\mathrm{DE}=$ Desviación estándar; $\mathrm{CV}=$ Coeficiente de variabibilidad 
den a presentar mayores longitudes de mecha (Quispe et al., 2008a [hembras: $3.10 \mathrm{~cm}$; machos: $3.06 \mathrm{~cm}$ ), mientras que en Abrapampa, Argentina es menor (Bustinza et al., 1995 [hembras: $2.98 \mathrm{~cm}$; machos: 2.59 $\mathrm{cm}]$ ).

A excepción del trabajo de Jahuira (1982), los estudios coinciden en indicar que el factor sexo no afecta la longitud de la mecha, lo cual se corrobora en el presente estudio. Probablemente, la ausencia de la expresión del dimorfismo sexual en las vicuñas es consecuencia de la precariedad, en cantidad y calidad, de la pradera altoandina y de la severidad climática (Quispe et al., 2016), los que en conjunto limitan no solo el aporte de nutrientes, sino ocasionan mayor gasto energético para contrarrestarla (San Martín, 1996). Por otro lado, los estudios evidencian que la longitud de mecha varía en función de factores tales como la localización espacial de las unidades de manejo, las condiciones agroecológicas de los hábitats que ocupan y la naturaleza biológica inherente a las subespecies en cada nicho ecológico (meridional y austral) (Wheeler et al., 2001).

Los promedios de longitud de mecha por clase se encuentran dentro de los rangos reportados. La mayor longitud de mecha de las vicuñas juveniles coincide con los registros de la Multicomunal Picotani, donde según Deza (1988), la longitud de mecha de las juveniles era de $3.09 \mathrm{~cm}$ y de las adultas era sde $3.03 \mathrm{~cm}$. Respecto al promedio regional, bajo condiciones de semicautiverio, las longitudes fueron ligeramente superiores a las reportadas por Aguilar (2009) para las clases juvenil $(3.27 \mathrm{~cm})$ y adulta $(2.56 \mathrm{~cm})$. Resaltan, en cambio, las vicuñas de Cala Cala por su mayor longitud de mecha (Bravo (1977) [cría: $3.49 \mathrm{~cm}$; juvenil: $3.39 \mathrm{~cm}$; adulto: 3.38 $\mathrm{cm}$ ]; Jahuira (1988) [juvenil: $4.76 \mathrm{~cm}$; adulta: $4.80 \mathrm{~cm}]$ ), aunque en años más recientes se ha reportado una diminución de la longitud de mecha en las juveniles $(2.84 \mathrm{~cm})$ (Montesinos, 2000).
Respecto a las vicuñas de la Sierra Central del país, las vicuñas juveniles de Pampa Galeras (2.37 cm; Montesinos, 2000) y de Huancavelica (3.01 cm; Quispe et al., 2008a) exhiben menor longitud de mecha; pero en el estudio de Quispe et al. (2008a,b), las adultas exhiben mayor longitud de mecha (3.15 $\mathrm{cm})$. En cambio, en Abrapampa, Argentina, las vicuñas juveniles resaltan por una mayor longitud de mecha $(4.61 \mathrm{~cm})$ en comparación con las adultas $(4.14 \mathrm{~cm})$.

Estos resultados evidencias dos eventos peculiares. El primero es que las fibras no crecen uniformemente desde que se originan en la piel, como consecuencia de la naturaleza de los grupos foliculares involucrados, pues los folículos pilosos se distribuyen sobre la piel en un arreglo característico, diferenciados en primarios y secundarios, y con distintas funciones (Bustinza, 2001). El segundo indica que el ritmo de crecimiento de la fibra de vicuña es lento. En virtud a ello, durante la época incaica, el Chacu se realizaba a intervalos de tres o más años (Koford, 1957), y en la actualidad la captura de las vicuñas si bien es anual, la esquila es bianual, o sea, no se esquilan a todos los animales capturados debido a que la fibra no alcanza la longitud mínima (Hoces, 2008).

\section{Longitud de Fibra}

La longitud de la fibra comprende el promedio de las mediciones individuales de cada una de las fibras. En sí, refleja la velocidad con que producen los bulbos pilosos de los folículos a través de la proliferación celular (Bustinza, 2001a). Asimismo, determina su uso textil.

Las mediciones individuales de las fibras permiten que se elongen en 33 y $36 \%$ respecto a su longitud de mecha en machos y hembras, respectivamente. No obstante, continúan por debajo del mínimo requerido para el proceso del peinado de la fibra (Villarroel, 1991). No se dispone de reportes sobre longitud de fibra a nivel regional, pero 
es evidente que las fibras de las vicuñas juveniles son más elongadas respecto a las vicuñas de Huancavelica (Zavaleta et al. (2009) [machos: $1.87 \mathrm{~cm}$; hembras: $1.90 \mathrm{~cm}$ ]; Quispe et al. (2014) [machos: (3.05 cm; hembras: $3.14 \mathrm{~cm}])$ y que las fibras de las vicuñas adultas (machos: $2.59 \mathrm{~cm}$; hembras: 2.94 $\mathrm{cm}$ ) y juveniles (machos: $3.20 \mathrm{~cm}$; hembras: $3.14 \mathrm{~cm}$ ) de Pampa Galeras, Ayacucho (Trejo y Rojas, 2009).

Los estudios, sobre la longitud de fibra, señalan que no hay diferencias estadísticas en función al factor sexo (Bravo, 1977; Montesinos, 2000), lo que corrobora el presente estudio. Los resultados reflejan que el crecimiento de la fibra de vicuña no es homogéneo debido a la localización espacial, a manera de parches, de los hábitats naturales que ocupan, producto de la cacería furtiva; los mismos que habrían inducido a los altos niveles de diferenciación biológica entre poblaciones (Wheeler et al., 2001).

En principio, la longitud de fibra muestra variaciones en función a la edad del animal; por otro lado, el elongamiento respecto a la longitud de mecha fue similar en crías y vicuñas juveniles (37\%), lo cual probablemente refleje el mayor grado de rizamiento al inicio de la vida de la vicuña, el cual disminuye con el avance de la edad a causa del engrosamiento propio de las fibras (Bustinza, 2001b; Quispe et al., 2016).

Las vicuñas juveniles y adultas de Huancavelica se caracterizan por tener una menor longitud de fibra (Quispe et al. (2014) [3.01, $3.16 \mathrm{~cm}$ ); Chanca (2011) [3.18 y 2.8 $\mathrm{cm}]$ ). Esto mismo, sucede en las vicuñas de Pampa Galeras (Lucanas, Ayacucho) (Trejo y Rojas (2009) [juveniles: 3.20 y $3.14 \mathrm{~cm}$; adultas: 2.59 y $2.94 \mathrm{~cm}$, en machos y hembras, respectivamente]); aunque Montesinos (2000) reportó valores superiores en vicuñas juveniles de Pampa Galeras $(4.40 \mathrm{~cm}$ ) y Cala Cala $(4.11 \mathrm{~cm})$. Por otro lado, Mueller et al. (2010) reportan $4.97 \mathrm{~cm}$ de longitud de fibra en vicuñas juveniles de Abrapampa, Argentina.
Los resultados podrían indicar que las vicuñas crías de la Multicomunal Picotani tienen la longitud de mecha mínima requerida por la industria textil; segundo, la longitud de mecha varía en función a la edad de la vicuña, estando la mayor expresión de la característica en la clase juvenil; tercero, el crecimiento de la fibra de vicuña es lenta y decrece con la edad del animal. A nivel general, el crecimiento de la fibra en la vicuña, expresada como longitud de mecha, está gobernada por factores de naturaleza hereditaria y ambiental (Carpio, 1991), disminuyendo a partir de la segunda esquila (Hoffman et al., 1983) o en forma progresiva a partir de los tres años (Bustinza et al., 1995), lo cual contrasta con el criterio comercial de lograr una mayor longitud para satisfacer las exigencias de la industria textil.

\section{Diámetro de Fibra}

El vellón está constituido por fibras finas y gruesas. Las primeras se concentran en la parte del lomo y flancos, mientras que las segundas se hallan mayormente en la región del pecho, las extremidades y la cara (Quispe et al., 2016). El diámetro o grosor o calibre o finura de la fibra determina la sensación de suavidad que se aprecia al tacto de los paños más finos y de valor (Carpio, 1991; Villarroel, 1991).

En términos generales, la finura de la fibra de vicuña muestra variaciones dentro y entre localidades del estudio para el factor sexo. Así, por ejemplo, resalta el diámetro de las vicuñas de Cala Cala, Puno (Jahuira (1982) [machos: $14.27 \mu \mathrm{m}$; hembras: $14.65 \mu \mathrm{m}$ ] 1982). Asimismo, Montesinos (2000) reporta para juveniles de Pampa Galeras (Ayacucho) diámetros de 13.55 y $14.50 \mu \mathrm{m}$ y en Cala Cala (Puno) de 13.92 y $14.53 \mu \mathrm{m}$, en machos y hembras, respectivamente, mientras que Nalvarte (1999) reporta para adultos de Pampa Galeras diámetros de 13.65 y $13.95 \mu \mathrm{m}$ y en Cala Cala de 14.80 y $14.96 \mu \mathrm{m}$, en machos y hembras, respectivamente. Años más tarde, Trejo y Rojas (2009) reportaron valo- 
res de 12.14 y $12.32 \mu \mathrm{m}$ en machos y hembras, respectivamente.

Similares resultados son reportados por Zavaleta et al. (2009) en vicuñas del CIPTT Tullpacancha (Huancavelica) (13.62 y $13.68 \mu \mathrm{m}$ en machos y hembras, respectivamente). Sin embargo, las vicuñas de la Multicomunal Picotani han mejorado su finura, ya que Deza (1988) reportó valores de 13.27 y $13.09 \mu \mathrm{m}$ en machos y hembras, respectivamente (Deza, 1988). Asimismo, presenta valores de finura menores al promedio regional (Aguilar, 2009 [Machos: $13.21 \mu \mathrm{m}$; hembras: $13.34 \mu \mathrm{m}$ ]).

Al parecer, la fibra se afina hacia el sur del área de estudio, pues en la fibra predescerdada de vicuñas del Parque Nacional Sajama, Bolivia, reporta 12.03 y $11.07 \mu \mathrm{m}$ en machos y hembras (Yapura, 2006), y 11.84 y $12.11 \mu \mathrm{m}$ en machos y hembras, respectivamente (Quizaya, 2007), mientras que hacia el norte (Huancavelica, Perú), la fibra presenta un mayor engrosamiento (Quispe $e t$ al., 2008a,b [machos: $12.78 \mu \mathrm{m}$; hembras: $13.16 \mu \mathrm{m}])$.

Con relación a la clase animal, la finura hallada se encuentra próxima al valor registrado a nivel de la Región Puno (Aguilar, 2009 [juveniles: $12.96 \mu \mathrm{m}$; adultas: $13.60 \mu \mathrm{m}$ ]), pero inferior al hallado en vicuñas del Parque Nacional de Sajama, Bolivia (Quizaya, 2007 [crías: 11.63 ìm; juveniles: 12.13 ìm; adultas: $12.18 \mathrm{im}])$. Sin embargo, resalta el diámetro de la fibra de las vicuñas de Cala Cala (Jahuira, 1982 [juvenil: $14.13 \mu \mathrm{m}$; adulta: 14.31 $\mu \mathrm{m}])$. Años más tarde, Montesinos (2000) reportó valores de 14.02 y $14.22 \mu \mathrm{m}$ en juveniles de Pampa Galeras y Cala Cala, respectivamente. No obstante, la finura de la fibra de las vicuñas de la Multicomunal Picotani se ha mantenido por años, considerando que Deza (1988) encontró $13.03 \mu \mathrm{m}$ en juveniles y $13.34 \mu \mathrm{m}$ en adultas.

En vicuñas de Huancavelica, Quispe et al. (2008b) reporta un diámetro de fibra de $12.53 \mu \mathrm{m}$ en juveniles y $13.41 \mu \mathrm{m}$ en adultas $(\mathrm{p}<0.01)$, y en Pampa Galeras, Trejo y Rojas
(2009) señalan en juveniles un valor de 11.25 $\mu \mathrm{m}$ y en adultos de $11.88 \mu \mathrm{m}$, sin diferencias significativas. Empero, estas variaciones se atribuyen a las formaciones demográficas a lo largo de Andes Centrales y del Sur y podrían representar grupos evolutivos (Wheeler et al., 2001).

Los resultados permiten reafirmar que la finura es el atributo más sobresaliente de la fibra de vicuña (Vilá, 2002).

\section{Conclusiones}

- La fibra de vicuña es corta, pero con una excepcional finura, sin que se manifieste un dimorfismo sexual.

- La longitud de mecha y fibra de las vicuñas juveniles es superior respecto a las crías y adultos.

\section{Literatura Citada}

1. Aguilar R. 2009. Influencia del sistema de manejo en el diámetro y longitud de fibra en vicuñas (Vicugna vicugna) en la Región Puno. Tesis de Médico Veterinario Zootecnista. Puno, Perú: Univ Nacional del Altiplano. $58 \mathrm{p}$.

2. Bravo MG 1977. Peso vivo, peso vellón, longitud de mecha y porcentaje de kemps y sus interrelaciones en vicuñas de Cala Cala. Tesis de Médico Veterinario Zootecnista. Puno, Perú: Univ Nacional del Altiplano. 59 p.

3. Bustinza V. 2001a. La alpaca. Conocimiento del gran potencial andino. Libro 1. Puno, Perú: Univ Nacional del Altiplano. $495 \mathrm{p}$.

4. Bustinza, V. 2001b. La alpaca. Crianza, manejo y mejoramiento. Libro 2. Puno, Perú: Univ Nacional del Altiplano. 343 p.

5. Bustinza V, Rebuffi G, Duga L. 1995. Producción de la vicuña en semicautividad. Abra Pampa. Argentina. En: Bioecología y uso sustentable de las 
poblaciones de vicuñas en la provincia de Jujuy-Argentina. Informe Estado de Avance 1995. Estación de Fauna Silvestre, Universidad Nacional de Jujuy, Argentina. $111 \mathrm{p}$.

6. Butler KL, Dolling M. 1995. Spinning fineness of wool. J Text Inst 85: 164166. doi: 10.1080/00405009508631319

7. Calle R. 1982. Producción y mejoramiento de la alpaca. Lima, Perú: Banco Agrario. $334 \mathrm{p}$.

8. Carpio M. 1991. La fibra de camélidos. En: Novoa C, Flores A (eds). Producción de rumiantes menores: Alpacas. Lima: RERUMEN. p 297-359.

9. Chanca G 2011. Determinación del diámetro de fibra de vicuña (Vicugna vicugna) y su correlación con el peso vivo. Tesis de Ing. Zootecnista. Huancavelica, Perú: Univ Nacional de Huancavelica. $68 \mathrm{p}$.

10. Deza A. 1988. Algunos parámetros del vellón de vicuñas de la SAIS Picotani Ltda. $\mathrm{N}^{\circ} 4$. Tesis de Médico Veterinario Zootecnista. Puno, Perú: Univ Nacional del Altiplano. $64 \mathrm{p}$.

11. [DGFFS] Dirección General de Forestal y Fauna Silvestre, Ministerio de Agricultura. 2012. Lineamientos técnicos para la conservación, manejo y aprovechamiento sostenible de los camélidos sudamericanos silvestres. Lima Perú: DGFFS. [Internet]. Disponible en: http://www.serfor.gob.pe/portal/ pdf/camelidos/lineaminetos_conservacion_camelidos.pdf

12. [DGFFS] Dirección General de Forestal y Fauna Silvestre, Ministerio de Agricultura. 2014. Censo poblacional de Vicuñas 2012. Lima Perú: DGFFS. 34 p.

13. Hoces D. 2008. Conservation and current use of the vicuña (Vicugna vicugna mensalis) in Peru. [Internet]. Disponible en: http://www.conabio.gob.mx/institucion/cooperacion internacional/TallerNDF/LinksDocumentos/WG-CS/WG5-Mammals/ WG5-CS8\%20Vicugna/WG5-CS8S.pdf
14. Hoffman R, Otte K, Ponce C, Ríos M. 1983. El manejo de la vicuña silvestre. Tomos I y II. Eschovborn-GTZ. 705 p.

15. IBNORCA] Instituto Boliviano de Normalización y Calidad. 1988. Norma Boliviana NB 953: Código recomendado de prácticas y criterios técnicos para el proceso de descerdado y clasificación de la fibra de camélido. Bolivia. [Internet]. Disponible en: http:// www.ibnorca.org/index.php/ normalizacion/desarrollo-de-normas

16. [INDECOPI] Instituto de Defensa del consumidor y de la Propiedad Intelectual. 2004. NTP 231.304 2004. Determinación de la longitud de mecha. Comisión de Reglamentos Técnicos y Comerciales - INDECOPI. Lima, Perú.

17. [INDECOPI] Instituto de Defensa del consumidor y de la Propiedad Intelectual. 2005. NTP 231.098 2005. Método para determinar el diámetro medio de la fibra de alpaca mediante el Microscopio de proyección. Comisión de Reglamentos Técnicos y Comerciales INDECOPI. Lima, Perú.

18. Jahuira F. 1982. Longitud de mecha, diámetro de fibra y rendimiento del vellón de vicuñas de Kala Kala. Tesis de Médico Veterinario Zootecnista. Puno, Perú: Univ Nacional del Altiplano. $57 \mathrm{p}$.

19. Koford C. 1957. The vicuna and the Puna. Ecological Monographs 27: 153-219.

20. Montesinos G 2000. Principales características del vellón de vicuñas juveniles a la primera esquila en Pampa Galeras y Cala Cala. Tesis de Médico Veterinario Zootecnista. Puno, Perú: Univ Nacional del Altiplano. $61 \mathrm{p}$.

21. Mueller JP, Rigalt F, Cancino AK, Lamas H. 2010. Calidad de las fibras de camélidos sudamericanos en Argentina. En: International Symposium on Fibers from South American Camelids. Conferencias Magistrales. Huancavelica, Perú.

22. Nalvarte J. 1999. Peso vivo, peso vellón, diámetro de fibra y porcentaje de pelos en vicuñas de Pampa Galeras y Cala Cala. Tesis de Médico Veterinario 
Zootecnista. Puno, Perú: Univ Nacional del Altiplano. $62 \mathrm{p}$.

23. Quispe EC, Paúcar R, Poma A, Sacchero D, Mueller JP. 2008a. Perfil del diámetro de fibras en alpacas. En: Proc Seminario Internacional de Biotecnología Aplicada en Camélidos Sudamericanos. Huancavelica. Perú.

24. Quispe DM. 2011. Análisis de la interacción entre las poblaciones de los animales domésticos y de vicuñas iestabilización o competencia? en la Reserva Nacional Pampa Galeras. Tesis de Maestría. Lima, Perú: Univ Nacional Agraria La Molina. $182 \mathrm{p}$.

25. Quispe E, Sánchez F, Filella J, Alfonso L. 2014b. Variation of commercially important characteristics among sampling sites for vicuña (Vicugna vicugna mensalis) fleeces. J Camelid Sci 7: 1-14.

26. Quispe E, Ramos H, Mayhua P, Alfonso L. 2008b. Fibre characteristics of vicuña (Vicugna vicugna mensalis). Small Ruminant Res. 93: 64-66. doi: 10.1016/j.smallrumres.2010.03.019

27. Quispe E, Rodríguez T, Iñiguez L, Mueller J. 2009. Producción de fibra de alpaca, llama, vicuña y guanaco en Sudamérica. Anim Genetic Resources 45: 1-14. doi:10.1017/S1014233909990277

28. Quispe JE, Apaza E, Quispe DM, Morocco N. 2016. De vuelta a la alpaca: producción primaria en una perspectiva empresarial y competitiva. Puno, Perú: Universidad Nacional del Altiplano. $444 \mathrm{p}$.

29. Quispe JE, Butrón B, Quispe DM, Chalco M. 2015. Producción de fibra de vicuña en semicautiverio y silvestria: tendencia, características y situación actual en la región Puno. Rev Investig Altoandin 17: 369-378.

30. Quizaya F. 2007. Estudio de la influencia del sexo y edad en la calidad de fibra pre-descerdada en vicuñas (Vicugna vicugna) del Parque Nacional de Sajama. Tesis de grado. Bolivia: Univ Mayor de San Andrés. 111 p.

31. San Martín F. 1996. Nutrición de camélidos sudamericanos y su relación con la reproducción. Rev Argentina Prod Anim 16: 305-312.

32. [SENAMHI] Servicio Nacional de Meteorología e Hidrología. 2012. Estación Meteorológica de Ananea. Puno, Perú.

33. Trejo W, Rojas E. 2009. Estudio tecnológico de la fibra y biometría de la vicuña (Vicugna vicugna) de la zona nuclear de Pampa Galera, LucanasAyacucho. Anales Científicos UNALM 70: 38-44. doi: 10.21704/ac.v70i1.70

34. Vilá B. 2002. La silvestría de las vicuñas, una característica esencial para su conservación y manejo. Ecol Austral 12: 78-92.

35. Villarroel J. 1991. Las fibras. En: Fernandez-Baca S (ed). Avances y perspectivas del conocimiento de los camélidos sudamericanos. Santiago de Chile: FAO. p 363-386.

36. Wheeler JC, Fernández M, Rosadio $R$, Hoces D, Kadwell M, Braford MW. 2001. Diversidad genética y manejo de poblaciones de vicuñas en el Peru. Rev Inv Vet Perú Supl 1: 170-183.

37. Yapura V, Copa S, Mamani E. 2006. Estudio de la calidad de la fibra de vicuña de vida silvestre en el parque nacional Sajama. Universidad Católica San Pablo. La Paz, Bolivia.

38. Zavaleta MJ, Quispe L, Baquerizo M. 2009. Características textiles de la fibra de vicuña (Vicugna vicugna) en el Centro de Investigación, Producción y Transferencia Tecnológica Tullpacancha - Huancavelica, Perú. Cien Des 14: 4550. doi: 10.21503/CienciayDesarrollo.2011.v14.04 